\title{
AN UNBOUNDED INVERSE PROPERTY IN THE ALGEBRA OF ABSOLUTELY CONVERGENT FOURIER SERIES ${ }^{1}$
}

\author{
JAMES D. STAFNEY
}

Let $A$ be the Banach algebra of complex valued functions $f$ on the circle of the form $f(x)=\sum c_{n} e^{i n x}$ where $\|f\|=\sum\left|c_{n}\right|$ is finite, $c_{n}$ is a complex number and the sums are taken over all the integers. A famous theorem due to Wiener asserts that if $f$ is in $A$ and is nowhere vanishing, then the reciprocal of $f$ is also in $A$.

The purpose of this note is to show that if a subset of $A$ consists of elements whose norms are bounded and whose ranges are uniformly bounded away from zero, then the norms of the inverses need not be bounded. This is the content of Theorem II. The proof of Theorem II rests heavily upon Theorem I, which is due to Katznelson [1]. A proof of Theorem I can be found in [2, p. 143].

We say that a complex valued function $F$ defined on $[-1,1]$ operates on $A$ in case $F(f(x))$ is an element of $A$ for each $f$ in $Q=\{f \in A$ : $\operatorname{sp}(f) \subset[-1,1]\}$. We use $\operatorname{sp}(f)$ to denote the range of $f$.

THEOREM I. If $F$ operates on $A$, then $F$ has an analytic extension to a neighborhood of $[-1,1]$ in the complex plane.

For positive real numbers $a, b, K$ with $a<b$, let $S(a, b, K)$ denote the set $\{f \in A: \operatorname{sp}(f) \subset[a, b]$ and $\|f\| \leqq K\}$.

TheOREM II. For some choice of positive real numbers $a, b, K$ with $a<b$, the set $\left\{\left\|f^{-1}\right\|: f \in S(a, b, K)\right\}$ is not bounded.

Proof. Assume that the theorem is false. Let $\epsilon_{n}$ be a sequence of positive real numbers such that $\sum \epsilon_{n}(n=1,2, \cdots)$ is finite. For $f$ in $Q,\left\|1+\epsilon_{n}-f\right\| \leqq 1+\epsilon_{n}+\|f\|$ and $\operatorname{sp}\left(1+\epsilon_{n}-f\right)$ is a subset of $\left[\epsilon_{n}, 2+\epsilon_{n}\right]$. Therefore, $1+\epsilon_{n}-f$ is in $S\left(\epsilon_{n}, 2+\epsilon_{n}, 1+\epsilon_{n}+\|f\|\right)$. For each positive integer $n$ choose $M_{n} \geqq 2^{n}$ so that $\left\|g^{-1}\right\| \leqq M_{n}$ forall $g$ in $S\left(\epsilon_{n}, 2+\epsilon_{n}, 1+\epsilon_{n}+n\right)$. Then

$$
\left\|\left(1+\epsilon_{n}-f\right)^{-1}\right\| \leqq M_{n}, \quad f \in Q, \quad\|f\| \leqq n .
$$

Define the function $F$ on $[-1,1]$ by

$$
F(t)=\sum_{n=1}^{\infty} \epsilon_{n} M_{n}^{-1}\left(1+\epsilon_{n}-t\right)^{-1}, \quad t \in[-1,1] .
$$

Received by the editors May 17,1966.

1 Partially supported by NSF grant GP-3509. 
For $f \in Q$,

$$
\left\|\epsilon_{n} M_{n}^{-1}\left(1+\epsilon_{n}-f\right)^{-1}\right\| \leqq \epsilon_{n}, \quad n \geqq\|f\| .
$$

Therefore, $F(f)$ is the limit in $A$ of the absolutely convergent series

$$
\sum_{n=1}^{\infty} \epsilon_{n} M_{n}^{-1}\left(1+\epsilon_{n}-f\right)^{-1}
$$

Since $f$ was arbitrarily chosen in $Q$, it follows that $F$ operates on $A$. But, by Theorem I, $F$ must have an analytic extension to a neighborhood of $[-1,1]$ in the complex plane; and this is impossible since $F$ on $[-1,1]$ is the restriction of a function meromorphic in the plane punctured at 1 with poles piling up there. This contradiction completes the proof of the theorem.

\section{REFERENCES}

1. Y. Katznelson, Sur les fonctions opérant sur l'algèbre des séries de Fourier absolument convergentes, C. R. Acad. Sci. Paris 247 (1958), 404-406.

2. Walter Rudin, Fourier analysis on groups, Interscience, New York, 1962.

YALE UNIVERSITY 